\title{
The Neural Modules Network with Collective Relearning for the Recognition of Diseases: Fault- Tolerant Structures and Reliability Assessment
}

\author{
Iraj Elyasi Komari ${ }^{1}$, Mykola Fedorenko ${ }^{2}$, Vyacheslav Kharchenko ${ }^{2}$, \\ Yevhenia Yehorova ${ }^{2}$, Nikolaos Bardis ${ }^{3}$ and Liudmyla Lutai ${ }^{2}$ \\ ${ }^{1}$ Islamic Azad University, Andimeshk, Iran \\ ${ }^{2}$ National Aerospace University KhAI, Kharkiv, Ukraine \\ ${ }^{3}$ Hellenic Army Academy, Athens, Greece
}

Received: September 4, 2020. Revised: November 17, $2020 \llbracket 6$ Accepted: November 18, 2020. Published: November 19, 2020.

\begin{abstract}
The article presents the architecture of multi-level information-analytical system (IAS) based on the neural modules network (NMN). This network consists of neural modules which are placed at the three levels (local, region and nation geographically distributed medical centers). Procedures of learning and collectiverelearning of neural modules consider region particularities and are based on analysis, generalization and exchange of experience related to diagnosis of diseases. These procedures provide modification and filtering parameters used as input for the further learning of local and regional neural modules.A few fault-tolerant structures of NMN-based IAS are researched taking into account different options of server and communication redundancy. Reliability block diagrams for redundant IAS structures are developed and formulas for calculation of probability of upstate are analyzed.
\end{abstract}

Keywords: neuro module network; medicalmulti-level information-analytical system; neural modules; diagnosing diseases; reliabilityinformation-analytical system;.

\section{INTRODUCTION}

$A$ $\mathrm{N}$ important task for medical IAS is the exchange and integration of the results of the diagnosis within the medical institutions at the regional and inter-regional (state) level. There are important regional factors influencing on the diseases.

To ensure trustworthy diagnosing diseases it is advisable to use neural modules (NM) and neuro module network (NMN) with the properties that contribute to the recognition of noisy or distorted objects. This allows for the collection and processing of the data obtained with the help of special software decision support systems, including by use of neural networks $[1-3]$.

Obviously, the correct diagnosis can be made as a result of taking into account all factors and features affect the decision on the diagnosis of diseases. To do this, health care facilities should be combined into a single information network of neural network diagnosis of diseases with a view to the timely exchange of experience [4-6]. At the same time it is very important to ensure the reliability and relevance of data sent and fault tolerance of NMN-based IASs in general $[7,8,9]$. In previous works $[10,11]$ structure such IAS and neural module for diagnosis of urologic diseases were described.

The paper [12] focuses on developing a comprehensive model to evaluate the impacts of Cyber-Physical Integration on distribution system reliability. This paper proposes a comprehensive model of distribution system reliability assessment considering cyber-physical integration. The minimal path method was employed to calculate reliability indices. Results of case studies indicate the impacts of Cyber-Physical Integration vulnerabilities as well as the features of Cyber-Physical Integration in smart distribution system.

Information exchange as part of shared decisionmaking is widely discussed in research and policy literature as means of improving patient involvement in treatment and care. The paper [13] is devoted to information exchange between patients and nurses for decision-making. The elements of information exchange within a recognized model of shared decision-making do not adequately fit with patient/nurse interactions in ward settings.

This study [14] suggests that urology program directors do not describe significant value to continued contact with applicants after the interview.

There is examined the decision support system for patient care using patient survey data in article. In particular, the patient is diagnosed with acute pain, urinary disorders, risk of infection of the urinary tract, impaired skin integrity, anxiety [15].

This article [16] proposed a reliable mechanism for processing and transmitting data, provided that there are maltifunctions in regional nodes.

The study assesses artificial neural networks contributions, compare performances and critiques methods The study found that neural-network models such as feedforward and feedback propagation artificial neural networks are performing better in its application to human problems [17].

Health care organizations are leveraging machinelearning techniques, such as artificial neural networks, to improve delivery of care at a reduced cost. Applications of 
artificial neural networks to diagnosis are well-known; however, ANN are increasingly used to inform health care management decisions [18].

The purpose of the paper [19] is to develop and research an availability model of a healthcare IoT system regarding failures of components. A detailed analysis of an architecture of healthcare IoT infrastructure is given. The main causes of the healthcare IoT based system failures are considered. Much attention is given to developing and research of a Markov model of a healthcare IoT system considering failures of components. Some essential highlevel requirements that such system must meet are presented. The analysis of obtained simulation results showed the rates that have the greatest influence on the availability function of the healthcare IoT system.

The goal of the paper is to present multi-level system for support of decision based on the network of neural modules and to develop and research reliability of a few fault-tolerant structures of the NMN-based IAS.

The paper is structured by the following way. The architecture and procedures of learning and collective relearning of neural modules are described in section II. Fault-tolerant structures of IAS and reliability models are suggested in section 3. Section 4 presents results of IAS reliability research and last section concludes the paper.

\section{SYSTEM}

\subsection{Architecture}

The multilevel hierarchical structure of NMN shown in Fig. 1 is a three-level tree diagram consisting of the following levels:

local, where the NM are geographically located directly in the medical institutions of the place of residence of patients in cities, towns, or district centers.

regional, which brings together all the local medical institutions of the region, and regional server is located in one of the medical institutions of the regional center.

- interregional (national) level, including a nationwide association of medical institutions of all regions, and the server corresponding to this level is located in one or more metropolitan medical centers.

The following tasks of IAS:

- the exchange of parameters being diagnosed interactively between medical centers;

the recognition of diseases taking into account the impact of regional environmental factors and individual characteristics of patients;

- conducting the operational (on-line) diagnosis of patients' diseases.

Detection of diseases in health centers is done by means of NM by processing the parameters obtained by means of technical diagnostics of organs or body systems functioning. The exchange of parameters being diagnosed is done by the use of training and additional training of NMN of patients' parameters with the same disease of related local, regional and inter-regional medical institutions.

Figure 2 is a block for support of the decision making (bdm) by doctor for the diagnosis of diseases of patients. In order to make decisions, the doctor receives input information in the form of a predicted diagnosis determined by a neural network module and additional patient examination data. In case of confirmation by the physician of the diagnosis, which is determined by the neural network module, the input and output are subsequently used for further training of other modules. In particular, local, regional and national levels.

\subsection{Learning and collective relearning}

A scheme of the doctor's processing of diagnostic information at the local level, where $\mathrm{x}_{\mathrm{j}, \mathrm{i}}$ - the input vector for the $i$-th NM in the $j$-th region, $y_{j, i}$ - its output value - form of the disease. Exchange of experience of disease recognition is carried out by using learning NM of diagnosed parameters of patients with the same diseases of related locals, regional and inter-regional medical institutions.

In the learning is used data with a 100 percent certainty indicating disease. Upon completion of learning the network is tested procedures to diagnose of diseases.

After the learning and testing the neural module network is used in the practice of diagnosing of diseases while in the case of confirmation of the diagnosis by the doctor which is determined NM, the input data are then used for relearning of other modules.

At the regional level, the learning of regional server is implemented upon receipt as learning data from a local neural modules of the region. Figure 3 presents a diagram of the interaction of local NMs with a regional server of the region, $p_{j}$ the number of $\mathrm{NMs}$ in the $\mathrm{j}$-th region, where $\mathrm{q}$ is the number of regions, $\mathrm{xr}_{\mathrm{j}}$ is the input, $\mathrm{yr}_{\mathrm{j}}$ is the output values of the $j$-th regional server. In the case of confirmation of the diagnosis by the doctor determined by NM, the input data are then used for relearning of local modules of the region.

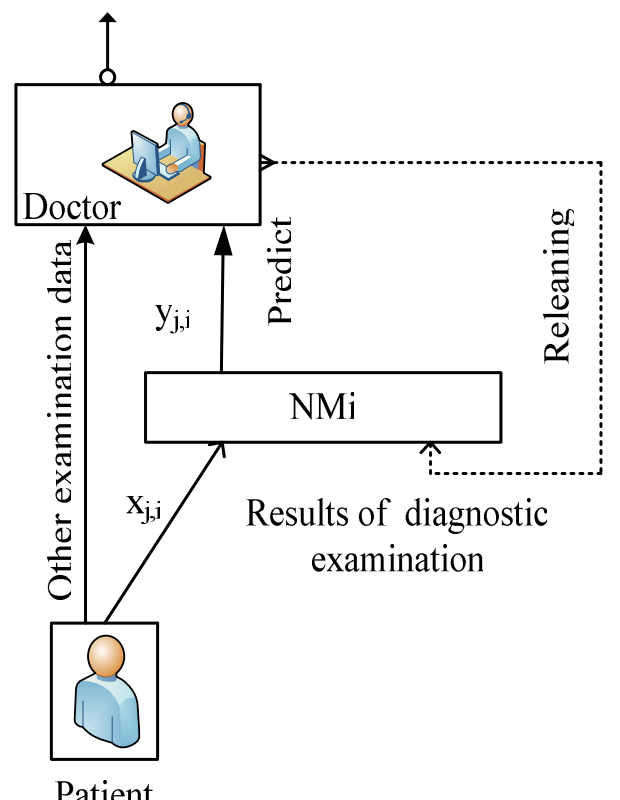

Figure 2 - Scheme of processing by the doctor of a diagnostic information of the patient(bdm) 


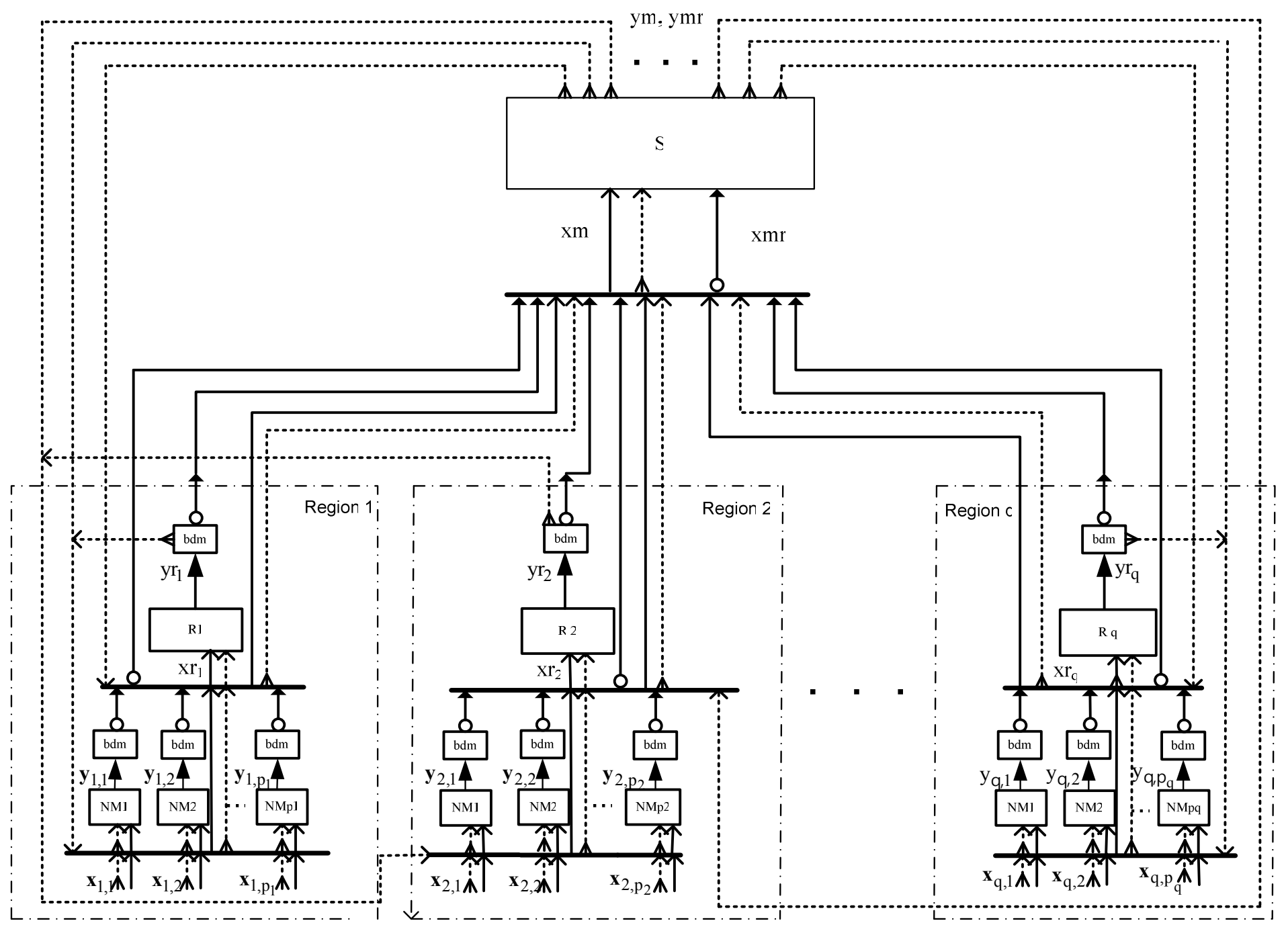

\section{Arrow purpose}

Input data for neural network training modules

$\longrightarrow$ Output data of neura network modules

$>-\cdots>\quad$ Input data relearning module

$\longrightarrow \quad$ Patient's diagnosis

\section{Legend:}

bdm - blok for support of the decision making by doctor

$\mathrm{S}$ - national server (national medical center neural network)

$\mathrm{Rj}$ - regional server ( Regional medical center neural network)

NMi - neural modules (local medical center neural network)

Figure 1 - Hierarchical processing of diagnostic information by neural modules network 


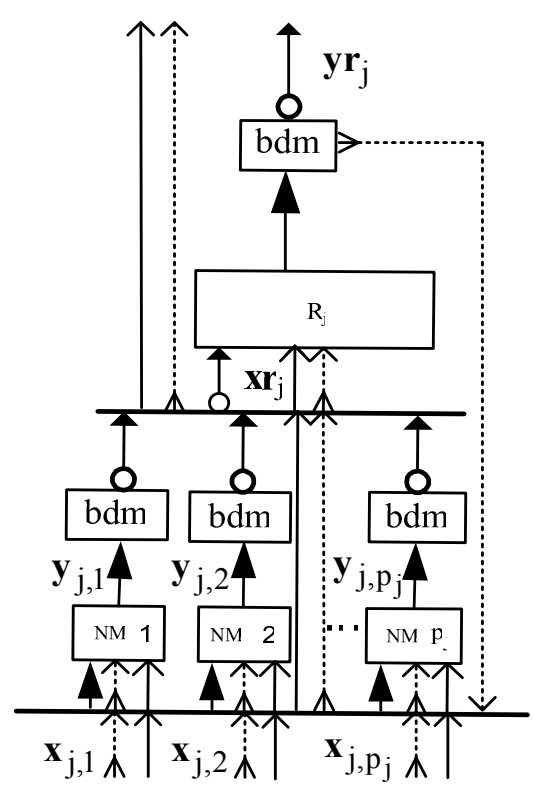

Figure 3 - Scheme of interaction between local NM and regional server

In the third interregional level the diagnostic conclusions, the output data of the local and regional levels, input data of local NM based on their regional location enter the input module.

At this stage the input and output data, their relationship with each other in order to select the most appropriate options for relearning of local NM and regional server are analyzed.

As the output data by the doctor's decision the network generates control actions $\mathrm{ym}, \mathrm{ymr}$ as learning data, accordingly for the local and regional levels. In fig. 4 a scheme of processing of the input data received from the local and regional medical institutions national server is shown.

National server performs one of three types of processing operations diagnosed parameters coming from local and regional medical centers by instructions of a doctor:

- relearning (a generalization of the experience of regional and local medical institutions) through learning and relearning of national server by the input data from local and regional medical institutions.

modification (selection). The NM is trained to handle the input data of the regional and local levels. At the same time the doctor can request the selection of the plurality of diagnosed parameters satisfying certain conditions and criteria.

- filtering (removal). Exclusion those data from the learning set which are unacceptable for certain NM of local or regional level.

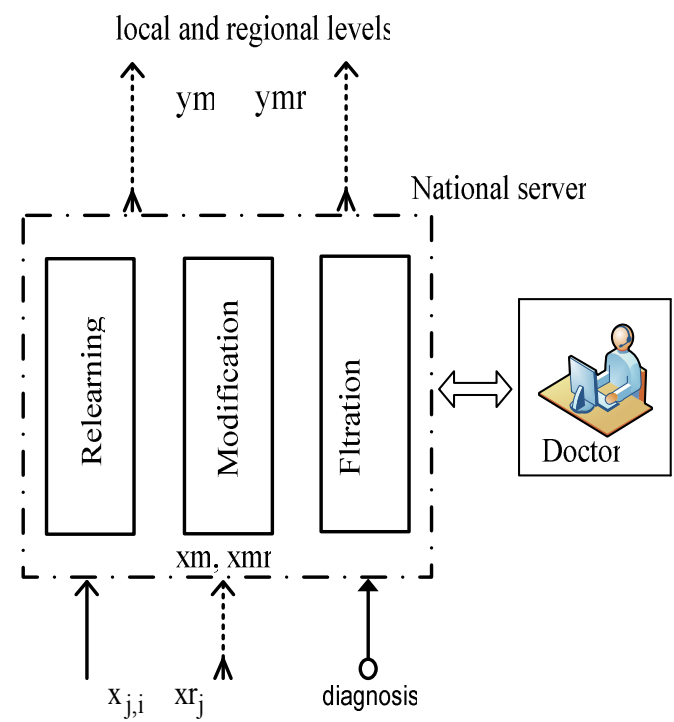

Figure 4 - Scheme of processing of the input data by national server

\subsection{Case study}

The previous sections provide an information system for the exchange of diagnostic information between health care facilities. At the local level, medical institutions conduct uroflowmetric examination of patients. According to the results of uroflowmetry, based on the use of NM, the obtained parameters are processed in order to diagnose the disease. In particular, the uroflowmetric parameter that influences decision making is the uroflowrogram schedule. In Figure 5 presents four types of uroflowrograms used to train the neural network module. The graphs of uroflowrograms correspond to the following disease classes:

- class №1 - insignificant infravesical obstruction;

- $\quad$ class №2 - sclerosis of neck of the urinary bladder;

- class №3 - schedule for patients with urethral strictures;

- class №4 - urination with detrusor sphincter dyssynergia.

As can be seen from the figure uroflowmetric graphs differ from each other. For class \# 1, the flow curve has an asymmetric appearance, and the maximum flow velocity is reached before the norm (10-15 seconds); class 2 is characterized by the fact that the maximum flow rate is reached later than normal; for class \# 3 the uroflowrogram has a "box" appearance without characteristic ups and downs; class No. 4 is characterized by small values of maximum and average flow velocity and as a consequence an increase in urination time [20].

The neural network of counter propagation was used as the neural network module of uroflourogramm identification.

To detect value of the maximum flow rate, depending on age, a neural network module was used as network with radial basis functions. The neural network generalizes the regression type to recognize the maximum and average speed values found in the shaded, lower part of the nomogram.

On the local level the diagnosis of urological disease is set, based on the processing of the results of neural network identification of uroflowmetric parameters and other data of the patient's survey. 
Further relearning of neural network modules for the diagnosis of diseases in urology based on the proposed approach is carried out using the following learning data derived from:

- Neural network of local units in the region;

- Regional server module;

- National server module.
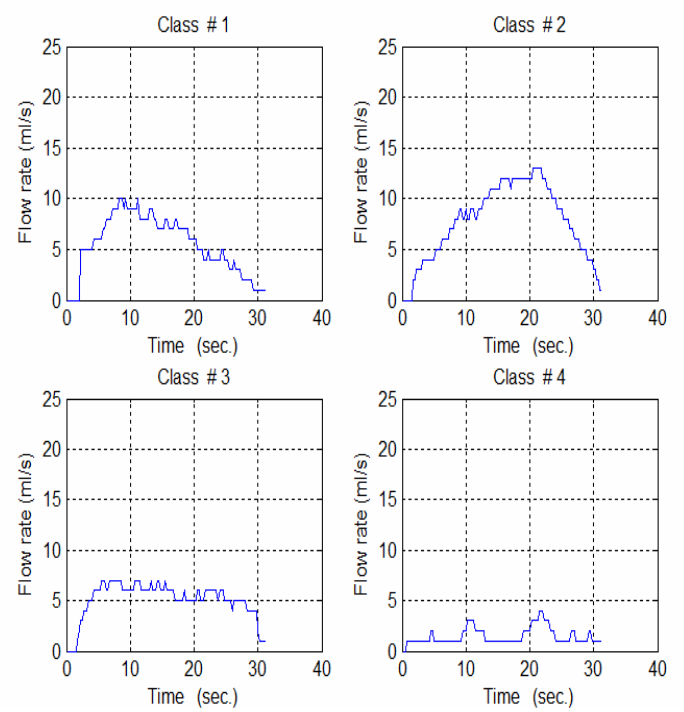

Figure 5 - Uroflourogramm charts of patients disease

Performing relearning operations, modifications, and filtration is carried out using national server.

Many diagnosed parameters are fed to the network input, and those parameters that meet the condition specified by the doctor are input to regional servers and local NMs.

\section{RELIABILITY MODELS}

Let's research reliability of different redundant of NMN based IAS structures basing figure 1 .

The NM are the simplest structural elements united by region. For each element in the system may be 2 conditions: the operation or the failure. All random events of failure free ware of elements are independent, namely, not affect the operability of the system as in whole.

The system consists of three levels. On the ground level there are NM connected in parallel. On the second level, these modules are grouped into regions, called the regional servers and each represents a $R_{i}, i=\overline{1, q}$. Regional server performs data processing and transfer it to the national level. The third level is the state (inter-regional unit) called the national server and designated as S. National server includes two components: communication and processing, referred to as $\mathrm{S}_{\mathrm{C}}$ and $\mathrm{S}_{\mathrm{D}}$. (see reliability block diagram (RBD) on Fig.6). The system is organized in such a way that in case of failure of any one region of the communication server takes over the functions of the failed region, and processing continues to receive data from the switching device. Consequently, only the server failure will lead to a malfunction.

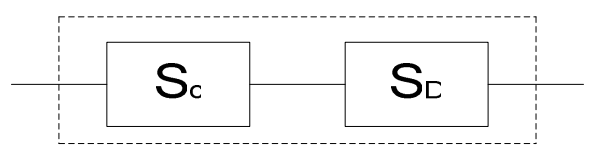

Figure $6-\mathrm{RBD}$ of national server

\subsection{The IAS structure with one national server}

The first option is the organization of the non-redundant system. RBD is shown in Figure 7. In the event of a server failure of the national system remains operational with limited functionality.

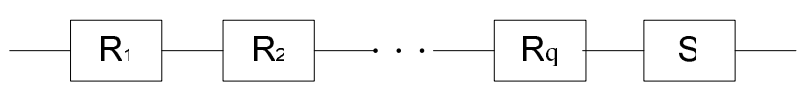

Figure $7-\mathrm{RBD}$ for system with limited functionality

The probability of failure-free operation of the system in this case:

$$
P_{0}=P_{S} \prod_{i=1}^{q} P_{R_{i}}
$$

where $\quad P_{0^{-}}$probability of failure-free of the system;

$P_{S^{-}}$probability of failure-free of the national

server; server.

$$
P_{R i}-\text { probability of failure of the i-th regional }
$$

If we assume that all $\mathrm{P}_{\mathrm{Ri}}$ have equal value, formula (1) will be the following:

$$
P_{0}=P_{S} P_{R}^{q}
$$

Bearing in mind that the server is composed of two components, the structural reliability of the system can be described as the circuit shown in Fig. 8. The system becomes operable in case of failure of the national server in general.

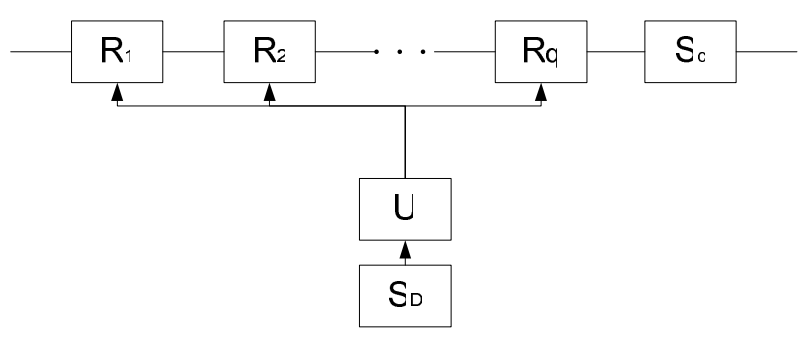

Figure $8-\mathrm{RBD}$ of the IAS with replacement of the regional servers by national server

If you add a clarification on the national server, then the probability of failure-free operation of the system in this case:

$$
\begin{gathered}
P_{0_{I}}=P_{S_{C}}\left[\prod_{i=1}^{q} P_{R_{i}}+P_{S_{D}} \sum_{i=1}^{q}\left(1-P_{R_{i}}\right) \prod_{j=1, j \neq i}^{q} P_{R_{j}}\right] \\
\text { if } P_{R_{1}}=\ldots=P_{R q}=P_{R},
\end{gathered}
$$

$$
P_{0_{I}}=P_{S_{C}}\left[P_{R}^{q}+q P_{S_{D}} P_{R}^{q-1}\left(1-P_{R}\right)\right]
$$


From this, it can be a platoon that on failure of at least one national server component system inoperable.

\subsection{The IAS structure with national server redundancy}

In the event of failure of one of the regional server system will continue to work with other regions, as moving the reservation is used. Therefore, any one regional server can be replaced with $S_{D}$ processing server. Inoperable system is only if all the regions are in a failed state or in case of failure of at least one national server component.

Therefore, the system must continue to work with other national duplicated processing server. That is, in terms of reliability in case of failure of one of the national servers handling the whole system remains operational. The condition of a system failure at this point is the simultaneous failure of both national server processing or communication server.

The RBD of the circuit will look like Fig.9.

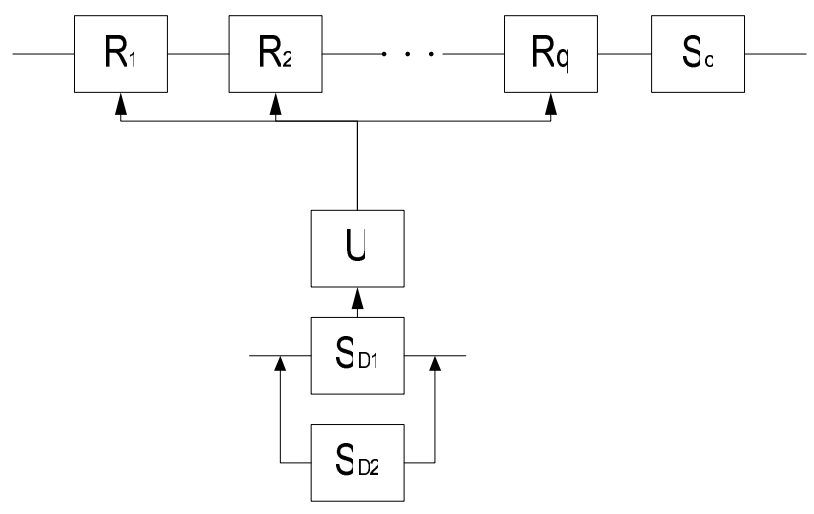

Figure 9 - RBD with national server redundancy

Thus, the system consisting of individual elements linked functionally so that an isolated failure of any one does not cause a system failure.

The probability of failure-free operation of the system in this case will be:

$$
\begin{gathered}
\text { if } P_{R_{1}}=\ldots=P_{R q}=P_{R} \\
P_{S_{D_{1}}}=P_{S_{D_{2}}}=P_{S_{D}} \\
P_{1_{I}}=P_{S_{C}}\left\{P_{R}^{q}+q P_{R}^{q-1}\left(1-P_{R}\right) *\left[P_{S_{D}}^{2}+2 P_{S_{D}}\left(1-P_{S_{D}}\right)\right]\right\}
\end{gathered}
$$

You can make the squad that in case of failure of the national processing server, the system remains operational while the works dubbed national processing server.

\subsection{The IAS structure with a few national servers}

In this embodiment, the block diagram of reliability regional servers grouped into two groups, each of which has its own national server processing, thereby reducing the load on the server. National communications server is duplicated. Therefore, in case of failure of one of the nation's system of processing servers will be partially operable, the system will be unusable only if denied all the regional server, or both national communications server. RBD shown in figure 10 .

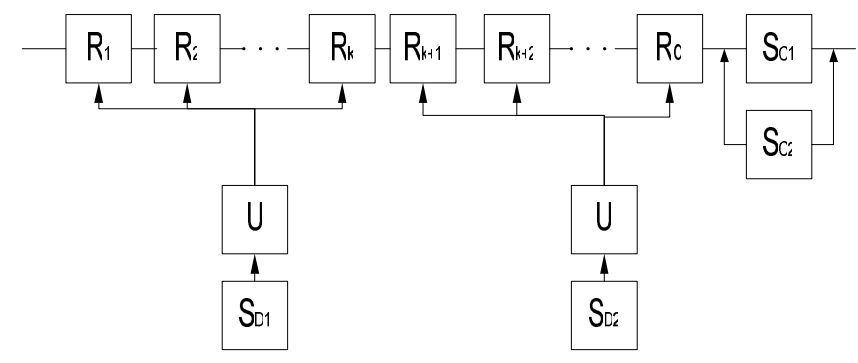

Figure $10-$ RBD with separate national servers

The probability of failure-free operation of the system will look like:

$$
\begin{gathered}
P_{2_{I}}=\left[\prod_{i=1}^{k} P_{R_{i}}+P_{S_{D_{1}}} \sum_{i=1}^{k}\left(1-P_{R_{i}}\right) \prod_{j=1, j \neq i}^{k} P_{R_{j}}\right] * \\
{\left[\prod_{i=k+1}^{q} P_{R_{i}}+P_{S_{D_{2}}} \sum_{i=k+1}^{q}\left(1-P_{R_{i}}\right) \prod_{j=k+1, j \neq i}^{q} P_{R_{j}}\right] *} \\
{\left[P_{S_{C_{1}}} P_{S_{C_{2}}}+\left(1-P_{S_{C_{1}}}\right) P_{S_{C_{2}}}+\left(1-P_{S_{C_{2}}}\right) P_{S_{C_{1}}}\right]} \\
\text { if } P_{R_{1}}=\ldots=P_{R q}=P_{R}, \\
P_{S_{D_{1}}}=P_{S_{D_{2}}}=P_{S_{D}}, \\
=\left[P_{R}^{k}+k P_{S_{D_{1}}} P_{R}^{k-1}\left(1-P_{R}\right)\right] \\
{\left[P_{R}^{q}+q P_{S_{D_{2}}} P_{R}^{q-1}\left(1-P_{R}\right)\right]} \\
{\left[2 P_{S_{C}}-P_{S_{D_{2}}}^{2}\right]}
\end{gathered}
$$

\section{RELIABILITY ANALYSIS}

After considering three options for the implementation of such a system, it is possible to analyze the probability of failure-free operation of the system, depending on the probability of failure of components.

If we take the input data $q=24$, and the probability of failure $\mathrm{P}_{\mathrm{Ri}}=0,9-0,99$. Probability of failure of the communication server $\mathrm{S}_{\mathrm{C}}=0,99$ and the likelihood of treatment failure server $S_{D}=0,99$. The probability of failure of the switching device $U=0$, we obtain the results of Table 1. Fig. 11 visualizes calculation results.

The graph clearly shows the advantage of the reliability of the model with separate national server.

If we take the input data $\mathrm{q}=24$, and the probability of failure $\mathrm{P}_{\mathrm{Ri}}=0,9-0,99$. Probability of failure of the communication server $\mathrm{S}_{\mathrm{C}}=0,99$ and the likelihood of treatment failure server $S_{D}=0,95$. The probability of failure of the switching device $U=0$, we obtain the results of Table 2.

Fig.12 visualizes calculation results. The graph clearly shows the advantage of the reliability of the model with separate national server.

If we take the input data $\mathrm{q}=24$, and the probability of failure $\mathrm{P}_{\mathrm{Ri}}=0,9-0,99$. Probability of failure of the communication server $\mathrm{S}_{\mathrm{C}}=0,95$ and the likelihood of treatment failure server $S_{D}=0,99$. The probability of failure 
of the switching device $U=0$, we obtain the results of Table 3.

Table 1.Probability of failure of the system depending on the probability of failure operation component

\begin{tabular}{|c|c|c|c|}
\hline $\mathbf{P}_{\mathbf{S D}}$ & \multicolumn{3}{|c|}{$\mathbf{0 . 9 9}$} \\
\hline $\mathbf{P}_{\mathbf{S C}}$ & \multicolumn{3}{|c|}{$\mathbf{0 . 9 9}$} \\
\hline $\mathbf{P}_{\mathbf{R}}$ & $\mathbf{P}_{\mathbf{0 I}}$ & $\mathbf{P}_{\mathbf{1 I}}$ & $\mathbf{P}_{\mathbf{2 I}}$ \\
\hline 0,9 & 0,2874 & 0,2895 & 0,4293 \\
\hline 0,91 & 0,3449 & 0,3473 & 0,4919 \\
\hline 0,92 & 0,4103 & 0,4131 & 0,5587 \\
\hline 0,93 & 0,4837 & 0,4868 & 0,6286 \\
\hline 0,94 & 0,5643 & 0,5677 & 0,7002 \\
\hline 0,95 & 0,6506 & 0,6542 & 0,7712 \\
\hline 0,96 & 0,7396 & 0,7433 & 0,8390 \\
\hline 0,97 & 0,8268 & 0,8303 & 0,9001 \\
\hline 0,98 & 0,9052 & 0,9082 & 0,9505 \\
\hline 0,99 & 0,9645 & 0,9664 & 0,9855 \\
\hline 1 & 0,9900 & 0,9900 & 0,9999 \\
\hline
\end{tabular}

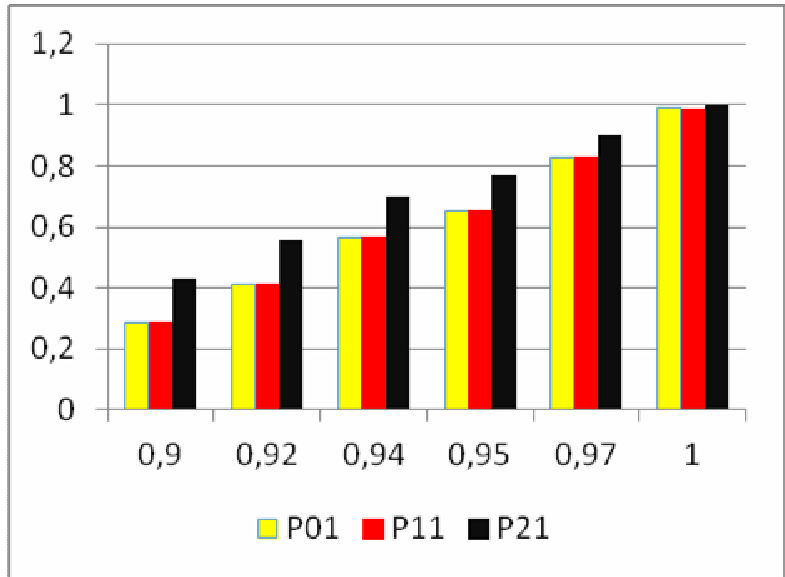

Figure 11 - Probability of failure of the system, depending on the probability of failure operation component

Table 2.Probability of failure of the system, depending on the probability of failure operation component

\begin{tabular}{|c|c|c|c|}
\hline $\mathbf{P}_{\mathbf{S D}}$ & \multicolumn{3}{|c|}{$\mathbf{0 . 9 5}$} \\
\hline $\mathbf{P}_{\mathbf{S C}}$ & \multicolumn{3}{|c|}{$\mathbf{0 . 9 9}$} \\
\hline $\mathbf{P}_{\mathbf{R}}$ & $\mathbf{P}_{\mathbf{0 I}}$ & $\mathbf{P}_{\mathbf{1 I}}$ & $\mathbf{P}_{\mathbf{2 I}}$ \\
\hline 0,9 & 0,2790 & 0,2890 & 0,4098 \\
\hline 0,91 & 0,3351 & 0,3467 & 0,4706 \\
\hline 0,92 & 0,3992 & 0,4124 & 0,5360 \\
\hline 0,93 & 0,4712 & 0,4861 & 0,6049 \\
\hline 0,94 & 0,5506 & 0,5669 & 0,6760 \\
\hline 0,95 & 0,6360 & 0,6533 & 0,7474 \\
\hline 0,96 & 0,7247 & 0,7424 & 0,8167 \\
\hline 0,97 & 0,8127 & 0,8295 & 0,8806 \\
\hline 0,98 & 0,8933 & 0,9075 & 0,9355 \\
\hline 0,99 & 0,9570 & 0,9659 & 0,9769 \\
\hline 1 & 0,9900 & 0,9900 & 0,9999 \\
\hline
\end{tabular}

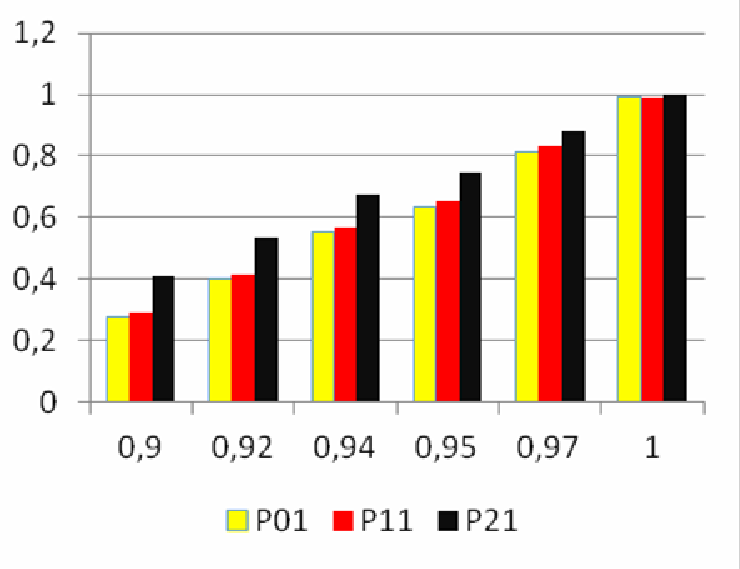

Figure 12 - Probability of failure of the system, depending on the probability of failure operation component

Fig.13 visualizes of calculation results.

The graph clearly shows the advantage of the reliability of the model with separate national servers.

Table 3.Probability of failure of the system, depending on the probability of failure operation component

\begin{tabular}{|c|c|c|c|}
\hline $\mathbf{P}_{\mathbf{S D}}$ & \multicolumn{3}{|c|}{$\mathbf{0 . 9 9}$} \\
\hline $\mathbf{P}_{\mathbf{S C}}$ & \multicolumn{3}{|c|}{$\mathbf{0 . 9 5}$} \\
\hline $\mathbf{P}_{\mathbf{R}}$ & $\mathbf{P}_{\mathbf{0 I}}$ & $\mathbf{P}_{\mathbf{1 I}}$ & $\mathbf{P}_{\mathbf{2 I}}$ \\
\hline 0,9 & 0,2790 & 0,2890 & 0,4098 \\
\hline 0,91 & 0,3351 & 0,3467 & 0,4706 \\
\hline 0,92 & 0,3992 & 0,4124 & 0,5360 \\
\hline 0,93 & 0,4712 & 0,4861 & 0,6049 \\
\hline 0,94 & 0,5506 & 0,5669 & 0,6760 \\
\hline 0,95 & 0,6360 & 0,6533 & 0,7474 \\
\hline 0,96 & 0,7247 & 0,7424 & 0,8167 \\
\hline 0,97 & 0,8127 & 0,8295 & 0,8806 \\
\hline 0,98 & 0,8933 & 0,9075 & 0,9355 \\
\hline 0,99 & 0,9570 & 0,9659 & 0,9769 \\
\hline 1 & 0,9900 & 0,9900 & 0,9999 \\
\hline
\end{tabular}

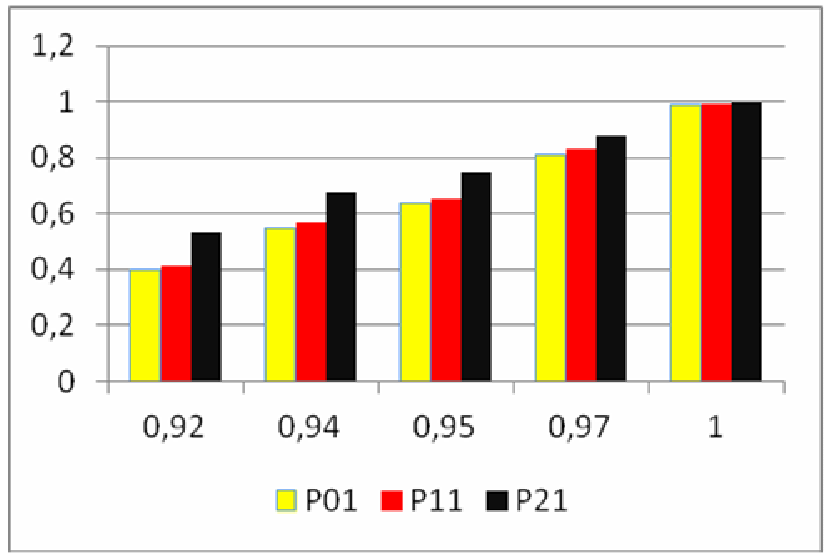

Figure 13 - Probability of failure of the system, depending on the probability of failure operation component

\section{CONCLUSIONS}

The paper presents a multilevel structure of neural network modules located on a territorial basis in specialized medical treatment and diagnostic facilities. This enables online diagnostic information to be exchanged for training and retraining of neural network modules. In order to 
enhance their generalization capabilities. For more accurate diagnosis results.

An approach to organizing training and retraining of neural network modules at each level of the hierarchical structure is proposed in order to take into account reliable patient diagnosis results. This allows, among other things, to take into account regional features of patients' diseases. At the national level, at the doctor's discretion, operations are performed to process diagnostic data. In particular, for regional and local modules, diagnostic data may be available for evaluation after modification or filtering. Such operations protect against false, incorrect, or unacceptable data.

The proposed models are validated on real patient diagnostic data. The uroflowmetric parameters that directly influence the diagnostic decision making are taken into account. In particular, to detect value of the maximum rate. The placement of points of maximum and average flow velocity on nomograms. The kind of a uroflowrogram schedule. This allows to obtain reliable data for diagnosing diseases. In addition, the intermediate states of the lower urinary tract are diagnosed. In particular, deviation from the norm and malfunction (obstruction). The models have been tested for four types of diseases.

A distinctive feature of the work is to address issues of reliability of data exchange between regional and national levels of IAS. A system consisting of individual elements and related functionally is proposed. The failure of any element does not cause the system to fail. We discussed three options for sharing diagnostic information models. For each option, RBD developed and presented analytical expressions for calculating the probability of failure-free operation of the system. Based on the data analyzed by the choice of a suitable variant of the model taking into account the failure probabilities of components and two components of the national server. The work calculates the probability of failure of the system on the proposed structures, depending on the probability of failure of components. Based on the above calculations, the structure with the separate national servers is the most reliable.

\section{References}

[1] W. Zhu, W. Huang, Z. Lin, Y. Yang, S. Huang, J. Zhou, "Data and feature mixed ensemble based extreme learning machine for medical object detection and segmentation", Multimedia Tools and Applications, Vol. 75, Issue 3, 2016.

[2] S. Belciug, F. Gorunescu, "Error-correction learning for artificial neural networks using the Bayesian paradigm. Application to automated medical diagnosis", Journal of Biomedical Informatics, Vol. 52, pp. 329-337, 2014.

[3] Johnsson, M., Garcia, Chamizo, J. M., Soriano Paya, A., Ruiz Fernandez, D. Application of artificial neural networks in the diagnosis of urological dysfunctions. Expert Systems with Applications, April 2009, vol. 36, iss. 3, part 2, pp. 5754-5760.

[4] G. Pearce , L. Mirtskhulava, K. Bakuria, J. Wong, S. Al-Majeed, N. Gulua, "Artificial Neural Network and Mobile Applications in Medical diagnosis", International Conference on Modelling and Simulation 17th UKSIM-AMSS, 2015, pp. 61 -65 .

[5] B. Banchev, T. Georgiev, "Architecture of automated communication and data processing system for forensic medicine", Proceedings of the 16th International Conference on Computer Systems and Technologies CompSysTech '15, 2015, pp. 160-167.

[6] A. Strielkina, V. Kharchenko, D. Uzun "Availability models for healthcare IoT systems: Classification and researchconsidering attacks on vulnerabilities “, Proceedings of 2018 IEEE 9th International Conference on Dependable Systems, Services and Technologies, Kyiv, Ukraine, $24-27$ May, 2018 (DESSERT2018), pp. 58-62.

[7] A. Avizienis, J.C. Laprie and B. Randell «Fundamental Concepts of Dependability», Research Report No 1145, LAAS-CNRS, April 2001.

[8] IEC 62628. Guidance on software aspects of dependability. Geneva: IEC, 2011, 63 p.

[9] V. A. Bogatyrev, A. V. Bogatyrev, "Functional Reliability of a Real-Time Redundant Computational Process in Cluster Architecture Systems "Automatic Control and Computer Sciences, Vol. 49, pp. 46 - 56, 2015.

[10] N. Fedorenko, V. Kharchenko, "Multilevel system for disease diagnosing based on neural network modules: principles of implementation", Radio electronic and computer systems, Vol. 6 (80), pp. 158-162, 2016.

[11] N. Fedorenko, V. Kharchenko, L. Lutay, Ye. Yehorova, "The processing of the diagnostic data in a medical information-analytical system using a network of neuro modules with relearning», IEEE East-West Design \& Test Symposium, pp. 381383, 2016.

[12] Hanjie Yuan, Gengfeng Li, Zhaohong Bie, Mariyam Arif, "Distribution System Reliability Assessment Considering Cyber-Physical Integration"Proceedings of the 10th International Conference on Applied Energy", Hong Kong, China, 22-25 August, 2018 (ICAE2018), Energy Procedia, Vol. 158, 2019, pp. 2655-2662.

[13] Vivianne Crispin, Carol Bugge, Kathleen Stoddart, "Sufficiency and relevance of information for inpatients in general ward settings: A qualitative exploration of information exchange between patients and nurses", International Journal of Nursing Studies, Vol. 75, pp. 112-122, 2017.

[14] Christopher J.Neylan, Arnav Srivastava, Shashank S. Pandya, Amy Kaplan, Kushan D. Radadia, Eric A. Singer, Sammy E. Elsamra, "The Urology Match Process and the Limited Value of Post-Interview Communication for Program Directors", Urology, Vol. 128, pp. 23-30, 2019.

[15] Angelica Te-Hui Hao, Lee-Pin Wu, Ajit Kumar, Wen-Shan Jian, Li-Fang Huang, Ching-Chiu Kao, Chien-Yeh Hsu, "Nursing process decision support system for urology ward", International Journal of Medical Informatics, Vol. 82, Issue 7, pp. 604-612, 2013.

[16] Sai Krishna Mothku, Rashmi Ranjan Rout, "Markov decision process and network coding for 
reliable data transmission in wireless sensor and actor networks", Pervasive and Mobile Computing, Vol. 56, pp. 29-44, 2019.

[17] Oludare Isaac Abiodun, AmanJantan, Abiodun Esther Omolara, Kemi Victoria Dada, Nachaat AbdElatif Mohamed, Humaira Arshad, "State-ofthe-art in artificial neural network applications: A survey“, Heliyon, Vol. 4, 2018, [Online]. Available:

https://doi.org/10.1016/j.heliyon.2018.e00938

[18] Nida Shahid, Tim Rappon, Whitney Berta,"Applications of artificial neural networks in health care organizational decision-making: A scoping review“،, PLOS ONE,Vol. 14 (2), 2019, [Online]. Available: https://journals.plos.org/plosone/article/file?id=10. 1371/journal.pone.0212356\&type $=$ printable

[19] A. Strielkina, V. Kharchenko, D. Uzun "A Markov Model of Healthcare Internet of Things System Considering Failures of Components", Proceedings of the 14th International Conference on ICT in Education, Research and Industrial Applications. Integration, Harmonization and Knowledge Transfer, Kyiv, Ukraine, 14 - 17 May, 2018 (ICTERI 2018), pp. 530-543.

[20] Lopatkin, N. A. Urologiya: natsional'noe rukovodstvo [Urology: national leadership]. Moscow, Izdatel'skaya gruppa GEOTAR - Media Publ., 2009. 1024 p.

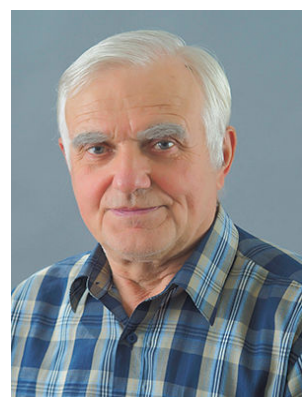

Mykola Fedorenko, Head of the Laboratory for Computer Technology and Multimedia Training at the Department of Applied Linguistics, National Aerospace University "KhAl" (Kharkiv, Ukraine). His scientific interests are intellectual information technology.

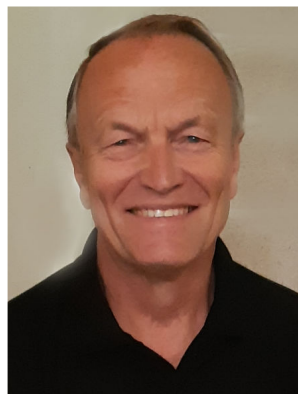

Vyacheslav Kharchenko, Dr of Science, Professor, is the Head of the Department of Computer Systems, Networks and Cyber Security at the National Aerospace University "KhAl" (Kharkiv, Ukraine) and the Head of STC, RPC Radiy. He has a Engineer Degreein Control Systems from Kharkiv High Rocket Engineering College, PhD Degree in Testing of Digital Systems in Military Academy of Rocket Troops, and DrS Degree in Dependable Systems from Kharkiv Military University. His scientific interests lay in area of the critical and green computing.

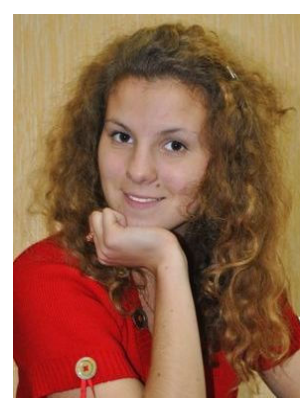

\section{Yevgeniya Yegorova,}

Department of Computer Systems, Networks and Cybersecurity, assistant.

Nikos Bardis, Eng., PhD., Associate Professor at the Hellenic Army Academy of the Department of Mathematics and

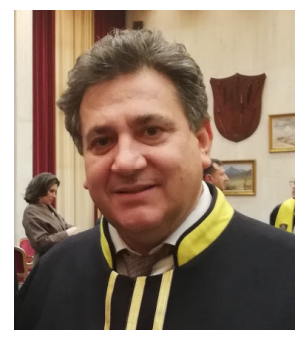
Engineering Sciences and Adjunct Prof. at the University of Athens Department of Mathematics. His research interests include Cryptography, Cyber Security, Systems Engineering and applications in Defence. He is Technical Program Committee (TPC) of the IEEE Societies (COMSOC), (TCCC), (TCSE).

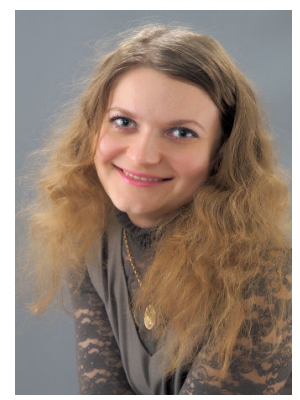

Liudmyla Lutai, PhD, is working at the National Aerospace University "KhAl" (Kharkiv, Ukraine). She is an associate professor. She has got a PhD degree in technical sciences. Her scientific interests lay in area of the Informational Technologies and artificial intelligence.

\section{Creative Commons Attribution License 4.0 (Attribution 4.0 International, CC BY 4.0)}

This article is published under the terms of the Creative Commons Attribution License 4.0 https://creativecommons.org/licenses/by/4.0/deed.en_US 